\title{
Comparision of the knee joint alignment in sportsmen and controls
}

\author{
HAAmaratunga ${ }^{1}$, MR Ileperuma ${ }^{2}$, W Rathnaweera ${ }^{3}$, SB Adikari ${ }^{1}$, HJ Suraweera ${ }^{2}$ \\ ${ }^{I}$ Department of Anatomy, Faculty of Medicine, University of Peradeniya, Peradeniya, Sri \\ Lanka, ${ }^{2}$ Orthopedic Unit Teaching Hospital Peradeniya, Sri Lanka, ${ }^{3}$ Teaching Hospital \\ Kegalle
}

Running title: knee alignment in rugger rites and controls

\begin{abstract}
The alignment of the knee joint described as the angle formed by the femoral shaft and the tibial shaft at the knee. It is of significance due to its association with different disease conditions affecting the knee. Muscle strength affects alignment of the knee, therefore we measured the knee alignment of Peradeniya University rugby team and compared with a control.
\end{abstract}

All 27 individuals in the university rugby pool, all of them being male, were included in the study. Their weight and height were measured in kilograms and meters using standard measuring scales and the BMI was calculated using the Quetelets index. Anterior plane knee alignment was measured using a handheld metal goniometer. The other group consisted of 25 male student volunteers that do not engage in sporting activities.

The rugby players had a mean knee alignment angle of $181.96^{\circ}( \pm 3.4)$ and the control sample had a mean of $183.67^{\circ}$ $( \pm 3.75)$. The difference observed in the means was statistically significant $(\mathrm{p}=0.45)$ with the rugby players' having more varus oriented knee joints.
The rugby players in our study showed a more varus oriented knee joint alignment than the controls. The varus orientation seen in these ruby players at this early age could be a cause for development of knee joint $\mathrm{OA}$ in the future due to the excessive strain placed on the medial compartment of the knee joint.

\section{Introduction}

The alignment of the knee joint described as the angle formed by the femoral shaft and the tibial shaft at the knee. It is of significance due to its association with different disease conditions affecting the knee (1). For example knee joint alignment is considered as a prognostic factor in both osteoarthritis and rheumatoid arthritis. Problems in the alignment: malalignment causes the weight distribution across the knee to change thereby causing undue stress on areas of the knee. This is seen commonly in knee osteoarthritis where mal-alignment of the knee towards the medial side causes increase in the severity of osteoarthritis of the medial compartment of the knee.

The factors that determine the alignment of the knee are many, being genetic factors, body mass index (BMI), laxity of the joint and muscle strength (2-4). While some of these factors maybe nonemodifiable, some such as BMI and muscle strength can be changed. 
HA Amaratunga, MR Ileperuma, W Rathnaweera, SB Adikari, HJ Suraweera - Comparision of the knee joint alignment in sportsmen and controls

Individuals engaged in sports on a regular basis generally have good muscle strength compared to an age matched sample that does not engage in sport regularly. Rugby is a sport which requires a great deal of muscle strength and those who engage in this sport have good lower limb muscle strength (5). However it is seen that some elite athletes and sportsmen tend to develop $\mathrm{OA}$ in later life and knee joint $\mathrm{OA}$ is the commonest condition (6). It is further stated that the overall muscle strength and fitness of the body prevents or decreases the rate of physical decline in such persons (6).

With this background it was the objective of this study to measure the knee joint alignment of Peradeniya University rugby team and compare with a group of students who do not engage in sporting activities on a regular basis in order to observe if alignment has a role to play in the development of OA in sportsmen.

\section{Materials and Methods}

The study was conducted at the Faculty of Medicine, University of Peradeniya. Ethical clearance was granted by the institutional review board, Faculty of Medicine, University of Peradeniya (No: 2008/EC/75). Informed, written consent was obtained from each participant.

All 27 individuals in the university rugby pool, all of them being male, were included in the study. Those with serious injuries to the hip knee or ankle and those with a history of surgery to the lower limb were excluded from the study. Their weight and height were measured in kilograms and meters using standard measuring scales and the BMI was calculated using the Quetelets index (7). Anterior plane knee alignment was measured using a handheld metal goniometer using the method described by Kraus et al (8). Readings were recorded up to 1 degree with the subject in an erect standing position, bare foot with toes placed forward and feet shoulder-width apart. The centre of the patella was located and marked with a pen. The center of the goniometer was placed on the center of the patella, and the arms of the goniometer were extended along the thigh visually bisecting it and along the axis of the lower leg along the first $4-5 \mathrm{~cm}$ of the subcutaneous border of the tibia in line with the middle of the ankle. The measurement was taken on both knees. Three consecutive measurements were taken by two separate investigators blinded to each other's findings. No significant inter or intra-observer variability occurred. The other group consisted of 25 male student volunteers that do not engage in sporting activities. This control sample was selected from a large sample (210) from a group of volunteers selected for a previous study (9). The weight, height and knee joint alignment of the controls were measured using the same instruments and methods.

\section{Results}

Sample from the rugby players consisted of 27 male individuals with an average age of $22.5 \pm 1.4$ years. The control sample consisted of 25 males with an average age of $23.07 \pm 0.95$ years. The 
HA Amaratunga, MR Ileperuma, W Rathnaweera, SB Adikari, HJ Suraweera - Comparision of the knee joint alignment in sportsmen and controls

mean BMI of the rugby players was $24.51( \pm 3.4)$ and that of the control group was $21.18( \pm 1.3)$. The difference in the mean BMI was statistically significant. The rugby players had a mean knee alignment angle of $181.96^{\circ}( \pm 3.4)$ and the control sample had a mean of $183.67^{\circ}$ $( \pm 3.75)$ (Figure 1). The difference observed in the means was statistically significant $(\mathrm{p}=0.45)$ with the rugby players' having more varus oriented knee joints.

\section{Discussion}

The rugby players in our study showed a more varus oriented knee joint alignment than the controls. An alignment study done on soccer players report similar findings in male soccer players and further report that athletes in general have more varus oriented knee joints (10). This is varus orientation is thought to be due to the greater strains that the knee is subjected to during sporting activities. It is interesting to see that those athletes who engage in sports that require lifting of heavy weights and building up of muscle strength have more varus oriented knees than those that require less of these attributes (10). The BMI of the rugby players was higher than the controls. BMI though a good measure of the body fat percentage is not a good indicator in instances where muscle mass is high as seen in athletes. The high BMI recorded in these rugby players is probably due to the higher muscle mass. However further evaluation of this measurement was beyond the scope of this study.
One limitation of this study is that the gold standard for the measurement of knee joint measurement was not used for this study. The gold standard being the angle measured on a full length anteroposterior (AP) weight bearing limb radiograph, which extends from the pelvis to the ankle joint (1). However this method exposes the participant to large amounts of radiation and cannot be utilized in a study of this nature. Kraus, et al., in 2005 concluded that knee alignment assessed clinically by goniometer correlated with the angle measured on the full-limb radiograph and that this alternative method was useful and appropriate in studies (8).

The varus orientation seen in these ruby players at this early age could be a cause for development of knee joint OA in the future due to the excessive strain placed on the medial compartment of the knee joint. However, long term follow-up and serial measurement of knee joint alignment is required to prove this association.

Dr(Mrs.) H.A. Amaratunga, Department of Anatomy, Faculty of Medicine, University of Peradeniya, Peradeniya, 20400, Sri Lanka

Email: himanipeiris@yahoo.com, himanip@pdn.ac.lk

Phone: 094812396275 , mobile 0773920177

Fax: 094812389106

Submitted by-April 2019

Accepted by - June 2019 
HA Amaratunga, MR Ileperuma, W Rathnaweera, SB Adikari, HJ Suraweera - Comparision of the knee joint alignment in sportsmen and controls

\section{References}

1. Moreland JR, Bassett LW, Hanker GJ. Radiographic analysis of the axial alignment of the lower extremity. J Bone Joint Surg Am. 1987;69: 745-9.

2. Bellemans J, Carpentier K, Vandenneucker $\mathrm{H}$, et al. The John Insall Award: Both morphotype and gender influence the shape of the knee in patients undergoing TKA. Clin Orthop Relat Res 2010;468:2936.

3. Fahlman L, Sangeorzan E, Chheda $\mathrm{N}$, et al. Older adults without radiographic knee osteoarthritis: knee alignment and knee range of motion. Clin Med Insights Arthritis Musculoskelet Disord 2014;7:1-11.

4. Moyer RF, Birmingham TB, Chesworth BM, Kean CO, Giffin RJ. Alignment, body mass and their interaction on dynamic knee joint load in patients with knee osteoarthritis. Osteoarthritis and Cartilage, 2010;18(7):888-893.

5. Comfort PP, Graham-Smith, et al. (2011). "Strength and power characteristics in English elite rugby league players." J Strength Cond Res 25(5): 1374-1384.
6. Guermaziae HM, Mezghanniae M, Ghroubiae S, Fkib H, Mefteha S, Bakloutic S, Sellamid S (2008) Knee osteoarthritis in 50 former top-level footballers: A comparative (control group) study. Annales de Réadaptation et de Médecine Physique, 51(3):174-178

7. Garrow JS and Webster JD (1985).Quetelets index as measurement of fatness. Int J Obes, 147.

8. Kraus VB, Vail TP, Worrell $\mathrm{T}$ and McDaniel G (2005) A comparative assessment of alignment angle of the knee by radiographic and physical examination methods, Arthritis Rheum.,52(6):1730-5

9. Amaratunga HA, Adikari SB, Dassanayaka TL, Chandrasekara M, Suraweera HJ. 2014.Goniometric alignment of the normal knee joint and the factors which affect it. Ceylon Med J 59: 45-48.

10. Colyn W, Agricola R, Arnout N, Verhaar J, Bellemans J (2016) How 
HA Amaratunga, MR Ileperuma, W Rathnaweera, SB Adikari, HJ Suraweera - Comparision of the knee joint alignment in sportsmen and controls

\section{Figures}

Figure1. Knee joint alignment in the rugby players and controls

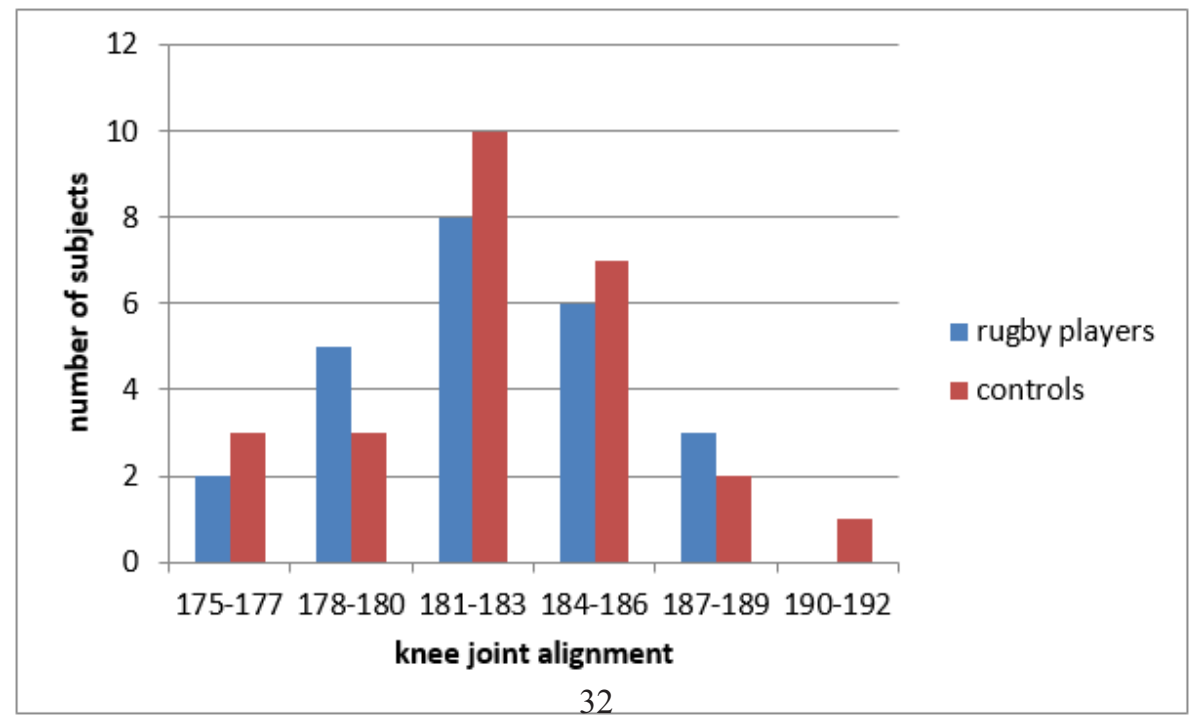

